\title{
Short-term variations in Be stars observed by the COROT and KEPLER space missions
}

\author{
Juan Gutiérrez-Soto ${ }^{1,2}$, Coralie Neiner ${ }^{2}$, Juan Fabregat ${ }^{3}$, Antonino $^{2}$ \\ Francesco Lanza ${ }^{4}$, Thierry Semaan ${ }^{5}$, Monica Rainer ${ }^{6}$ and \\ Ennio Poretti ${ }^{6}$ \\ ${ }^{1}$ Instituto de Astrofísica de Andalucía (CSIC), Glorieta de la Astronomía s/n 18008, \\ Granada, Spain; email: jgs@iaa.es \\ ${ }^{2}$ LESIA, Observatoire de Paris, CNRS, Université Paris Diderot, \\ 5 place Jules Janssen, 92190 Meudon, France \\ ${ }^{3}$ Observatorio Astronómico de la Universidad de Valencia, \\ Calle Catedrático Agustín Escardino 7, 46980 Paterna, Valencia, Spain \\ ${ }^{4}$ INAF - Osservatorio Astrofisico di Catania, via S. Sofia, 78, 95123 Catania, Italy \\ ${ }^{5}$ GEPI, Observatoire de Paris, CNRS, Université Paris Diderot, \\ 5 place Jules Janssen, 92190 Meudon, France \\ ${ }^{6}$ INAF - Osservatorio Astronomico di Brera, via E. Bianchi 46, 23807 Merate (LC), Italy
}

\begin{abstract}
The COROT and KEPLER space missions are collecting very high-precision longduration photometric data of many Be stars, allowing us to better understand the origin of their short-term variability and the link between these variations and the Be phenomenon. In this paper, we present a brief summary of the results obtained in the analysis of several Be stars observed with COROT in terms of pulsations. In addition, we show that variations of the Be star HD 175869 can be explained as two active regions separated by 150 degrees or as unstable pulsating modes in a star with an extensive mixing in radiative layers corresponding to a core overshooting of $0.35 \mathrm{Hp}$. A preliminary study of the photometric and spectroscopic variability seen in the B1.5IVe star HD 51193 is performed. Currently the KEPLER satellite is observing the only confirmed Be star in its field of view, namely KIC 6954726. From low-resolution spectra we derived a spectral type of B2.5Ve for this star and we studied the long-term variation of the emission in the $\mathrm{H} \alpha$ line. The 3.5-year KEPLER light curve will allow us to detect even more close frequencies than with COROT and to perform a detailed analysis of the amplitude variations in a Be star.
\end{abstract}

Keywords. stars: oscillations, stars: emission-line, Be

\section{Introduction}

Be stars are main sequence or slightly evolved B-type stars with emission in the Balmer lines arising from a circumstellar disk. It is generally agreed that the disk is fed by episodic mass ejections (outbursts) but the mechanism at work is yet uncertain. Be stars are very fast rotating stars, with an average angular velocity of $90 \%$ of the critical breakup velocity (Frémat et al. 2005). Therefore, an additional mechanism is needed to provide the angular momentum to eject material into the circumstellar disk. Osaki in 1986 proposed nonradial pulsations as the mechanism to produce these outbursts. More recently, Rivinius et al. (1998) found spectroscopically that the mass-loss episodes in the star $\mu$ Cen could be explained by the beating of pulsating modes. A correlation between outbursts and pulsations was also found photometrically in two stars observed with COROT (Huat et al. 2009, see below; Gutiérrez-Soto et al. 2010). Alternatively, Balona (2009) suggested that the eruptions could be of magnetic origin and proposed a simple model for Be stars based 
Table 1. Overview of the first Be stars that were observed with the COROT satellite. In the colums, we give for each object: the HD number, spectral type, inclination angle in ${ }^{\circ}$ (with an error bar of $10^{\circ}$ for all stars), $v \sin i$ (in $\mathrm{km} \mathrm{s}^{-1}$ ), rotation frequency, the main low and high frequencies (in $d^{-1}$ ) detected with СОROT, the frequencies detected in line-profile variations, and the references.

\begin{tabular}{|c|c|c|c|c|c|c|c|c|}
\hline Star & SpT & $i$ & $v \sin i$ & $f_{\text {rot }}$ & low freq. & high freq. & LPV & Ref \\
\hline HD 49330 & B 0.5IVe & 40 & $280 \pm 10$ & $0.96 \pm 0.35$ & $1.47,2.94,0.87$ & $11.86,16.89$ & $11.86,16.89,1.51$ & {$[1],[2]$} \\
HD 51193 & B1.5IV & 37 & $220 \pm 25$ & $0.89 \pm 0.3$ & $1.40,0.72,2.62,1.78$ & no & $1.78,1.39,0.71$ & {$[6]$} \\
HD 181231 & B5IVe & 45 & $190 \pm 25$ & $0.76 \pm 0.32$ & $0.62,0.69,1.24$ & no & 0.69 & {$[5]$} \\
HD 175869 & B8IIIe & 50 & $170 \pm 10$ & $0.64 \pm 0.1$ & $0.64,1.28$ & no & no & {$[4]$} \\
HD 50209 & B8IVe & 60 & $200 \pm 20$ & $0.62 \pm 0.2$ & $1.48,2.16,0.79,0.67$ & no & no & {$[3]$} \\
\hline
\end{tabular}

[1] Huat et al. (2009), [2] Floquet et al. (2009), [3] Diago et al. (2009), [4] Gutiérrez-Soto et al. (2009), [5] Neiner et al. (2009), [6] Frémat et al. (2006) and this work.

on magnetic fields. However, no clear detection of magnetic fields in Be stars has been found so far.

Be stars show different timescales of variability in their light curves and spectra: from weeks to decades, caused by changes in the envelope; and from hours to days, produced by non-radial pulsations or/and rotational modulation. A long discussion was held during the last decade about the origin of these short-term variations (Baade \& Balona 1994).

From an exhaustive spectroscopic campaign of 27 early-type Be stars, Rivinius et al. (2003) showed that the short-term periodic line profile variability (LPV) of these objects is due to non-radial pulsations. The LPV of the majority of stars in the sample were identified with the mode $\ell=m=2$. More recently, the MOsT satellite observed $4 \mathrm{Oe} / \mathrm{Be}$ stars during 30-40 consecutive days and suggested that non-radial pulsations are involved in all rapidly rotating Be stars (see Cameron et al. 2008 and references therein). COROT has shown that most of the observed Be stars are multiperiodic and that pulsations are present in early to late Be stars (see below for more details).

Alternatively, Balona (1995) found a correlation between the photometric periods and their projected rotational velocities and proposed that short-term variability in Be stars is caused by stellar spots or co-rotating clouds attached to the star by a magnetic field. Again this model assumes the presence of a stellar magnetic field and no detection has been obtained so far.

\section{Overview of the COROT results}

COROT consists of a space telescope dedicated to stellar seismology and the search for extrasolar planets. It is observing up to 150 consecutive days of the same fields with a precision of a few $\mu$ mag and a data sampling of 32s (Auvergne et al. 2009). For a summary of the first results, see Michel et al. (2008).

\subsection{COROT observations}

In Table 1 a short summary of the results obtained for the first Be stars observed with COROT is given. $f_{\text {rot }}$ was taken from the Tables in each reference, assuming that the rotational velocity is at $90 \%$ of the critical velocity.

The earliest Be star HD 49330 was found to be a hybrid Be star showing p- and gmodes (Huat et al. 2009). The amplitudes of the p- and g-modes varies significantly along the COROT observations. An outburst occured just after the amplitude of the two main g-modes were at maximum and while the amplitude of the p-modes had drastically decreased. After the outburst the amplitude of the g-modes decreased and the p-modes recovered a larger amplitude. This showed, for the first time in photometry, a clear 


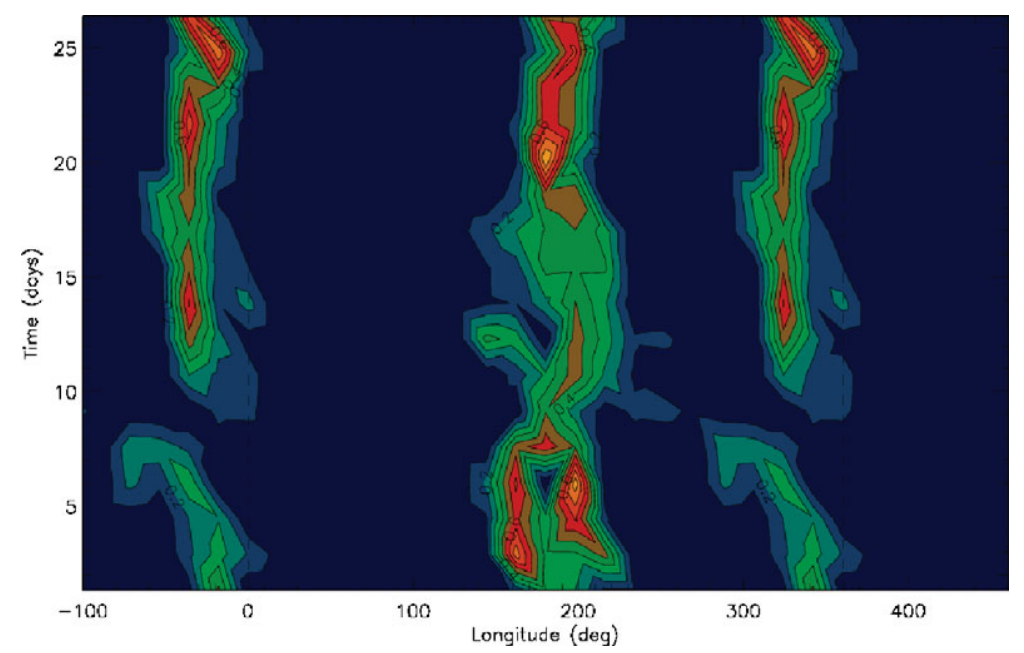

Figure 1. Isocontour plot of the filling factor versus longitude and time for the star HD 175869. Two regions are always active and separated by 150 degrees.

correlation between pulsations and outbursts. In addition, thanks to the study of spectroscopic time series we could identify the degree of the p-modes $(\ell \sim 4$ and 6$)$.

The other early-type Be star, HD 51193, only shows g-modes in its light-curve and several of them could be identified in spectroscopy as well. See details below.

Multiple g-mode frequencies were detected in the COROT light curve of the mid-type Be stars HD 181231 (Neiner et al. 2009). One of the frequency is also detected in spectroscopy.

The 26-day light curve of the late-type star HD 175869 is dominated by a smallamplitude frequency and its harmonics (Gutiérrez-Soto et al. 2009). These variations are interpreted as inhomogeneities at the surface of the star, although non-radial pulsations are not excluded (see below in Sect.2.2).

Multiple periods due to g-modes have been detected in the late-type Be star HD 50209 (Diago et al. 2009). In addition, for the first time, the frequencies showed a clear pattern produced by the rotational splitting, which is not expected by models for such a rapidly rotating star (it rotates at least at $90 \%$ of the critical angular velocity). This star does not show a double wave phase diagram.

\subsection{Modelling the star HD 175869}

Non-radial pulsations could explain the variations seen in the COROT light curve of the star HD 175869. Applying pulsating models, as in Saio et al. (2007), we found unstable g-modes that are close to the observed frequencies. However, the star should be in the core-hydrogen burning stage elongated by an extensive mixing in the radiative layers corresponding to a core overshooting of $0.35 \mathrm{Hp}$. See Neiner et al. (2010), Lovekin et al. (2010).

As the light curve of the star HD 175869 is a double-wave with a period compatible with the rotational period, these variations could also be explained as spots or inhomogeneities in or close to the photosphere that rotate with the star. In order to confirm/reject this hypothesis, we applied the Maximum Entropy method (Lanza et al. 2009) to model the light curve as spots. The light curve was divided into 17 subsets of duration of the assumed rotational period (1.56 days, $0.64 d^{-1}$ ) and each one was modelled independently from the others, giving us the possibility to trace spot evolution one rotation after the other. 


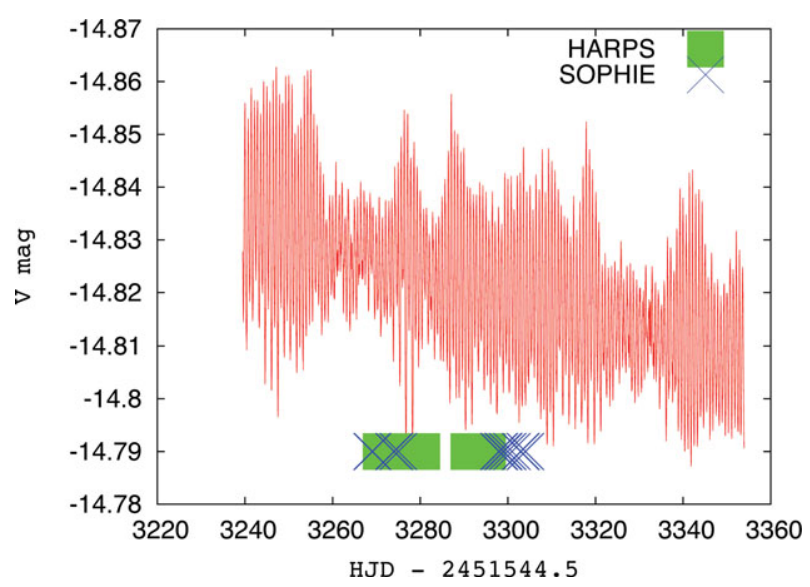

Figure 2. COROT light curve of the star HD 51193. The time of observations with HARPS and SOPHIE are indicated.

The residuals of this modelling are of the order of the residuals of the fitting given in Gutiérrez-Soto et al. (2009).

The first result is that there are two main active longitudes, where individual spots form and evolve. They are separated by about 150 degrees and have comparable levels of activity as measured by the spot filling factors (see Fig. 1). We would expect a separation of 180 degrees between the active regions if the spots are produced by a dipole magnetic field. However, if the dipole magnetic field is offcentered, i.e. if there is a quadrupole component, the regions where the spots would be attached may be separated by less than 180 degrees. The results will be published in a forthcoming paper (Gutiérrez-Soto, Lanza et al. 2010).

\section{New results}

\subsection{HD 51193}

The star HD 51193 was observed in the seismology fields of COROT during 114 days (LRa2 run). The light curve, depicted in Fig. 2, shows a clear beating, also seen in most of the Be stars observed with COROT. A dominant peak is found in the periodogram at $1.399 d^{-1}$ with an amplitude of $15 \mathrm{mmag}$. Many frequencies close to this one are detected with amplitudes ranging from 5 to $0.7 \mathrm{mmag}$, which could be produced by amplitude changes. Other peaks were found at frequencies $0.721 d^{-1}(\sim 3 \mathrm{mmag}), 0.156 d^{-1}$ ( $\sim 2 \mathrm{mmag}), 2.617 d^{-1}$ ( 1 $\left.\mathrm{mmag}\right)$, and $2.798 d^{-1}$ ( 1mmag). No clear combination is found among these frequencies, except for the frequency $2.798 d^{-1}$ which is twice $1.399 d^{-1}$. No frequencies higher than $5 d^{-1}$ are found in the periodogram of this earlytype Be star.

High-resolution spectra were obtained with HARPS at the 3.6-m telescope in La Silla (PI E. Poretti, ESO LP 182.D-0356) and with SOPHIE at the $1.93 \mathrm{~m}$ telescope at the OHP in December 2008/January 2009 (PI P. Mathias). They were collected simultaneously with the COROT data, as can be seen in Fig. 2.

A preliminary study of the line-profile variations reveals several frequencies at 1.78 , 1.40 and $0.71 d^{-1}$ with coherent phase distributions across the line-profile. In Fig. 3 we display the power and phase distributions across the HeI 4388 line for the frequencies 1.78 and $0.71 d^{-1}$. Note the different slope of the phase for each frequencies, suggesting a different degree or azimuthal order of the mode. 

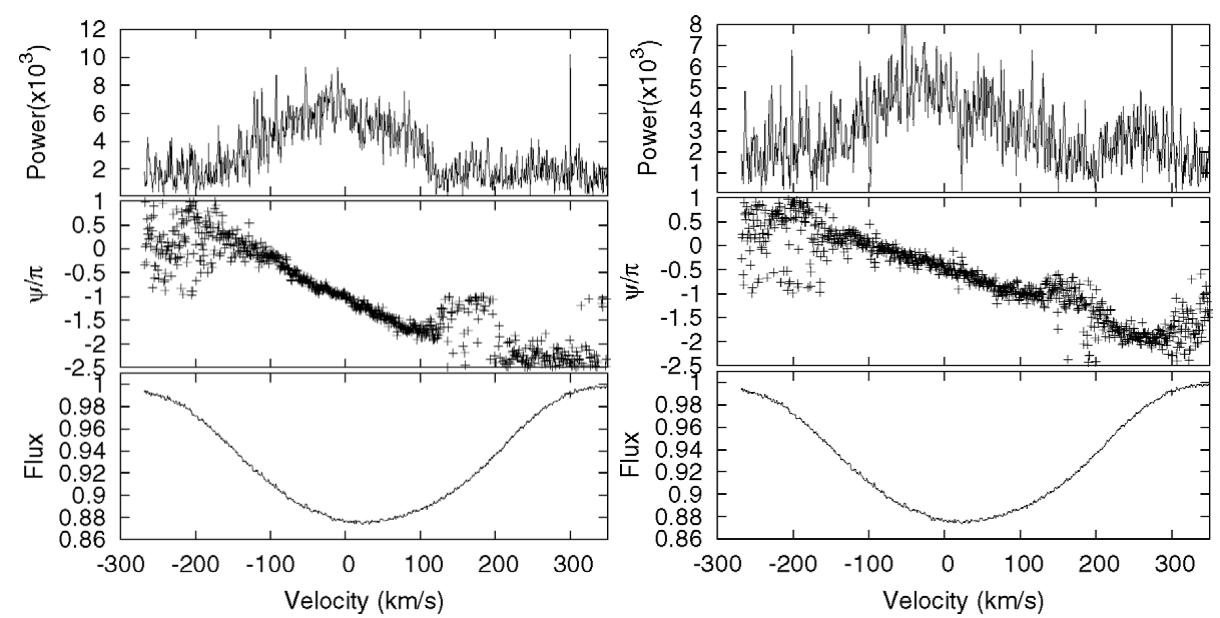

Figure 3. Power distribution (top) and phase distribution (middle) across the He I 4388 line profile (bottom) for $\mathrm{f}=1.78$ (left) and $0.71 d^{-1}$ (right), for the star HD 51193 .

The multiple frequencies detected in the COROT light curve and the spectroscopic time series are attributed to non-radial pulsations.

\subsection{KEPLER: a promising future}

The KEPLER mission (Koch et al. 2010) is designed to detect Earth-like planets around solar-type stars by the transit method. KEPLER is continuously monitoring the brightness of over 100000 stars for at least $3.5 \mathrm{yr}$ in a 105-square degree fixed field of view.

The only confirmed Be star being observed by KEPLER is the star KIC 6954726. The low-resolution spectra obtained during the last 2 years suggest that this star is a B2.5Ve. In addition, we have followed the variation of its $\mathrm{H} \alpha$ line: it is always in emission and its equivalent width has been increasing since 2008 .

The longer duration of the KEPLER observations with respect to COROT runs (typically 150 days) will give us the opportunity to distinguish between intrinsic amplitude variations produced by transient frequencies or amplitude variations due to the beating of very close frequencies. Hopefully, this long duration will also allow us to observe an outburst with KEPLER.

\section{Conclusions}

Some questions have arised from these studies. Can we explain the multiple frequencies found in most of Be stars observed with COROT and MOST with stellar spots or co-rotating clouds? The observed frequencies are coherent and independent and are often numerous, which would require a high number of independant spots i.e. a complex magnetic field. In addition, note that no clear detection of a magnetic field in a Be star has been obtained so far. Non-radial pulsations thus seem a simpler explanation and pulsation models are very successful in reproducing observations. On the other hand, can we explain the amplitude variations and double-wave shape seen in many of the COROT Be stars with non-radial pulsations?

All these questions will hopefully be answered with the help of the space missions COROT, KEPLER and later on PLATO. In addition, non-perturbative methods (eg. Ballot et al. 2010) are needed to model the non-radial pulsations in these rapidly rotating Be stars: they will give us the keys to understand these observations. 


\section{Acknowledgements}

J. Gutiérrez-Soto acknowledges PNPS and the IAU for awarding him a grant to participate to the symposium. We wish to thank the COROT team for the acquisition and reduction of the COROT data. The HARPS data was obtained as part of the ESO Large Programme LP182.D-0356 (PI: E. Poretti). This research is also based on spectroscopic data from the SOPHIE spectrograph at OHP (France).

\section{References}

Auvergne, M., Bodin, P., Boisnard, L., \& Buey, J.-T. et al. 2009, A\&A, 506, 411

Baade, D., Balona, L. A. 1994, in: L. A. Balona, H. F. Henrichs, \& J. M. Le Contel (eds.), Pulsation; Rotation; and Mass Loss in Early-Type Stars, IAU Symposium 162, p. 311

Balona, L. A. 1995, MNRAS, 277, 1547

Balona, L. 2009, in: J. A. Guzik, \& P. A. Bradley (eds.), Stellar Pulsation: Challenges for theory and observation, AIP Conf. Ser., 1170, p. 339

Ballot, J., Lignières, F., Reese, D. R., \& Rieutord, M. 2010, A\&A, 518A, 30

Cameron, C., Saio, H., Kuschnig, R., Walker, \& G. A. H. et al. 2008, ApJ, 685, 489

Diago, P. D., Gutiérrez-Soto, J., Auvergne, M., \& Fabregat, J. et al. 2009, A\&ऽA, 506, 125

Floquet, M., Hubert, A.-M., Huat, A.-L., \& Frémat, Y. et al. 2009, A\&A A, 506, 103

Frémat, Y., Zorec, J., Hubert, A.-M., \& Floquet, M. 2005, A $\& A$ A, 440, 305

Frémat, Y., Neiner, C., Hubert, A.-M., \& Floquet, M. et al. 2006, A\&A, 451, 1053

Gutiérrez-Soto, J., Floquet, M., Samadi, R., \& Neiner, C. et al. 2009, A\&A, 506, 133

Gutiérrez-Soto, J., Semaan, T., Garrido, R., \& Baudin, F. et al. 2010, AN, in press, arXiv: 1010.1910

Gutiérrez-Soto, J., Lanza, A. F. et al. 2010, A\&SA in preparation

Huat, A.-L., Hubert, A.-M., Baudin, F., \& Floquet, M. et al. 2009, A\&A, 506, 95

Koch, D. G., Borucki, W. J., Basri, G., \& Batalha, N. M. et al. 2010, ApJ (Letters), 713, L79

Lanza, A. F., Pagano, I., Leto, G., \& Messina, S. et al. 2009, A\&A A, 493, 193

Lovekin, C.C., Neiner, C., Saio, H., \& Mathis, S. et al. 2010, AN, in press

Michel, E., Baglin, A., Weiss, W. W., \& Auvergne, M. et al. 2008, Communications in Asteroseismology 156, 73

Neiner, C., Gutiérrez-Soto, J., Baudin, F., \& de Batz, B. et al. 2009, A\& A, 506, 143

Neiner, C., Mathis, S., Saio, H., \& Lovekin, C. et al. 2010, A\&SA in preparation

Osaki, Y. 1986, PASP, 98, 30

Rivinius, T., Baade, D., Stefl, S., et al. 1998, in: L. Kaper \& A.W. Fullerton (eds.), Cyclical Variability in Stellar Winds, ESO Astrophysics Symposia (Berlin, New York: SpringerVerlag), p. 207

Rivinius, T., Baade, D., \& Štefl, S. 2003, A\&̈A 411, 229

Saio, H., Cameron, C., Kuschnig, R., \& Walker, G. A. H. et al. 2007, ApJ, 654, 544

\section{Discussion}

M. Sмiтh: Unfortunately, it appears that there are some difficulties about observing Be stars with KEPLER. First, the difficulty to find B stars with the KEPLER photometric catalogue: it was dispensed with their " $u$ " filter of the original filter set from the sloan survey and therefore, the KEPLER project's ground-based photometry does not allow to distinguish between a mid O star and an O star. Second, the fixed KEPLER field of view is centered several degrees from the Galactic plane. Furthermore, KEPLER cannot easily observe bright stars at all because of constraints in downlinking and transmitting data to the Earth. For these reasons the observable OB stars that one will likely find will be intrinsically luminous or "runaway" stars from the Galactic plane. One can hope to be lucky and perhaps find a good variable or Be candidates. 